TRANSACTIONS OF THE

AMERICAN MATHEMATICAL SOCIETY

Volume 353, Number 11, Pages 4567-4581

S 0002-9947(01)02836-7

Article electronically published on June 1, 2001

\title{
UNBOUNDED COMPONENTS OF THE SINGULAR SET OF THE DISTANCE FUNCTION IN $\mathbb{R}^{n}$
}

\author{
PIERMARCO CANNARSA AND ROBERTO PEIRONE
}

\begin{abstract}
Given a closed set $F \subseteq \mathbb{R}^{n}$, the set $\Sigma_{F}$ of all points at which the metric projection onto $F$ is multi-valued is nonempty if and only if $F$ is nonconvex. The authors analyze such a set, characterizing the unbounded connected components of $\Sigma_{F}$. For $F$ compact, the existence of an asymptote for any unbounded component of $\Sigma_{F}$ is obtained.
\end{abstract}

\section{INTRODUCTION}

Motzkin's Theorem in convex analysis states that a nonempty closed set $F \subseteq \mathbb{R}^{n}$ is convex if and only if the projection onto $F$ of any point $x \in \mathbb{R}^{n}, \operatorname{proj}_{F}(x)$, is a singleton. This is one of the simplest examples that show how properties of $\operatorname{proj}_{F}$ can be used to describe properties of $F$.

In this paper we are interested in the set of all points $x \in \mathbb{R}^{n}$ at which $\operatorname{proj}_{F}$ is multivalued, hereafter denoted by $\Sigma_{F}$. As is well-known, an equivalent definition of $\Sigma_{F}$ can be given in terms of the euclidean distance function $d_{F}$-another well-known object. In fact, the set on which $\operatorname{proj}_{F}$ is multivalued coincides with the set of all points $x \in \mathbb{R}^{n} \backslash F$ at which $d_{F}$ fails to be differentiable, see, e.g., [5] p. 62]. We refer to the latter set as the singular set of $d_{F}$. The above characterization of $\Sigma_{F}$ as the singular set of $d_{F}$ is very useful as it provides simple proofs of rather fine measure theoretic estimates. For example, since $d_{F}$ is Lipschitz continuous, Rademacher's Theorem implies that $\Sigma_{F}$ has Lebesgue measure zero. It is noteworthy that this result was proved by Erdös [4] by a completely different method.

The local structure of the singular set has been investigated by several authors in view of its importance for best approximation theory. For instance, Bartke and Berens 3 proved that any non-isolated singular point of $d_{F}$ is the initial point of a Lipschitz singular arc - a result formerly obtained by Pauc [8] for $n=2$. This analysis was later extended to Hilbert spaces by Westphal and Frerking [10], see also 9].

The first object of our analysis is the global structure of the connected components of $\Sigma_{F}$. In particular, we are interested in classifying the connected components of $\Sigma_{F}$ according to their boundedness properties. We observe that, for a simply connected compact set $F$ that is not convex, the unboundedness of $\Sigma_{F}$ is ensured by another classical result of Motzkin's [7. Our approach uses the one-toone correspondence between the connected components of the set $\overline{\mathrm{co}} F \backslash F$ and the

Received by the editors October 19, 2000 and, in revised form, December 20, 2000

1991 Mathematics Subject Classification. Primary 41A65, 26A27; Secondary 34A60, 49J52.

Key words and phrases. Distance function, metric projection, best approximation, singularities, differential inclusions.

(C)2001 American Mathematical Society 
components of $\Sigma_{F}$ that was introduced by Veselý in [9]. Denoting by $\mathcal{M}$ such a correspondence, in Theorem 3.1 we show that a component $C$ of $\Sigma_{F}$ is bounded if and only if $\mathcal{M}^{-1}(C)$ is bounded and is also a component of the set $\mathbb{R}^{n} \backslash F$. In particular, whenever $F$ fails to contain the topological boundary of $\overline{\mathrm{co}} F$, the existence of an unbounded connected component of $\Sigma_{F}$ follows.

Once the unbounded components of $\Sigma_{F}$ have been characterized, we turn our attention to the asymptotic behaviour of such components. More precisely, for any $n \geq 2$ and for $F$ compact we show that an unbounded component $C$ of $\Sigma_{F}$ always possesses an asymptotic half-line, in the sense that $d_{C}\left(x_{0}+t v\right) \rightarrow 0$ as $t \rightarrow+\infty$ for some $x_{0}, v \in \mathbb{R}^{n}$ with $\|v\|=1$. Also, for $n=2$, we prove that the above convergence to the asymptote takes a stronger form. In fact, in this case, we construct a continuous arc, $\gamma$, contained in $C$ and asymptotic to the half-line $x_{0}+t v$, and we show that $\gamma$ coincides - up to reparametrization - with the unique absolutely continuous solution of

$$
\left\{\begin{array}{l}
\xi^{\prime}(s) \in D^{+} d_{F}(\xi(s)), \quad \text { a.e. } s \geq 0, \\
\xi(0)=\bar{x}
\end{array}\right.
$$

(here $\bar{x}$ is a fixed point of $C$ ). Notice that inclusion (1.1) has already been used in the literature to study the local structure of $\Sigma_{F}$ (see [3] and [1]).

This paper is organized as follows. In section 2 we recall basic notions on the distance function and on the singular set of the metric projection. Section 3 is devoted to the characterization of the unbounded connected components of $\Sigma_{F}$. In section 4 we prove the existence of an asymptote for general $n$, and then we improve this result in section 5 for $n=2$.

\section{Notation and Preliminary Results}

For any nonempty subset $A$ of $\mathbb{R}^{n}$, we denote by $\mathcal{P}(A)$ the family of all subsets of $A$, and by $\mathcal{C}(A)$ the set of all connected components of $A$.

We denote by $x \cdot y$ the scalar product between two vectors $x, y \in \mathbb{R}^{n}$, and by $\|x\|$ the usual euclidean norm of $x$. The canonical basis of $\mathbb{R}^{n}$ will be denoted by $e_{1}, \ldots, e_{n}$ and we shall set, as usual, $x_{k}=x \cdot e_{k}$. Given $x, y \in \mathbb{R}^{n}$ we denote by $[x, y]$ the line segment

$$
[x, y]=\{t x+(1-t) y: t \in[0,1]\} .
$$

For any $x \in \mathbb{R}^{n}$ and any $r>0$ we set

$$
B(x, r)=\left\{y \in \mathbb{R}^{n}:\|y-x\|<r\right\}
$$

and we abbreviate $B(r)=B(0, r)$.

In this paper, $F$ stands for a nonempty closed subset of $\mathbb{R}^{n}$. Moreover, we denote by co $F$ the convex hull of $F$ and by $\overline{\operatorname{co}} F$ the closure of co $F$.

The distance function from a closed set $F, d_{F}$, is defined as

$$
d_{F}(x)=\min _{y \in F}\|x-y\| \quad \forall x \in \mathbb{R}^{n} .
$$

For any $\eta>0$ we set $F_{\eta}=\left\{x \in \mathbb{R}^{n}: d_{F}(x)<\eta\right\}$. The set of closest points in $F$ to a given point $x \in \mathbb{R}^{n}$ will be denoted by

$$
\operatorname{proj}_{F}(x)=\left\{y \in F: d_{F}(x)=\|x-y\|\right\} .
$$


We shall also refer to $\operatorname{proj}_{F}(x)$ as the (euclidean) projection of $x$ onto $F$. The following is an elementary property of the distance function that we need for the sequel. We give a proof for the reader's convenience.

Lemma 2.1. Let $\xi:] a, b\left[\rightarrow \mathbb{R}^{n}\right.$ be a continuous arc such that $\xi(t) \notin F$ for any $t \in] a, b\left[\right.$, and define $f(t)=d_{F}(\xi(t))$. If $\xi$ and $f$ are both differentiable at some point $\bar{t} \in] a, b[$, then

$$
f^{\prime}(\bar{t})=\xi^{\prime}(\bar{t}) \cdot \frac{\xi(\bar{t})-x}{\|\xi(\bar{t})-x\|} \quad \forall x \in \operatorname{proj}_{F}(\xi(\bar{t})) .
$$

Proof. Let $x \in \operatorname{proj}_{F}(\xi(\bar{t}))$. Observing that $f(t) \leq\|\xi(t)-x\|$ for any $\left.t \in\right] a, b[$ and $f(\bar{t})=\|\xi(\bar{t})-x\|$, we obtain

$$
\frac{f(t)-f(\bar{t})}{t-\bar{t}} \leq \frac{\|\xi(t)-x\|-\|\xi(\bar{t})-x\|}{t-\bar{t}} \quad \forall t>\bar{t}
$$

Hence, taking the limit as $t \downarrow \bar{t}$,

$$
f^{\prime}(\bar{t}) \leq \xi^{\prime}(\bar{t}) \cdot \frac{\xi(\bar{t})-x}{\|\xi(\bar{t})-x\|} .
$$

The opposite inequality is easily obtained arguing similarly for $t<\bar{t}$.

We denote by $\Sigma_{F}$ the singular set of $d_{F}$, that is the set of all points $x \in \mathbb{R}^{n} \backslash F$ at which $d_{F}$ fails to be differentiable. As observed in the Introduction, $\Sigma_{F}$ has Lebesgue measure zero. More is actually true. In fact, the representation formula

$$
d_{F}^{2}(x)-\|x\|^{2}=\min _{y \in F}\left(\|x-y\|^{2}-\|x\|^{2}\right)=\min _{y \in F}\left(\|y\|^{2}-2 x \cdot y\right)
$$

implies that $x \mapsto d_{F}^{2}(x)-\|x\|^{2}$ is concave. So, by known results for singular sets of concave functions (see, e.g., [1] and [2]), $\Sigma_{F}$ can be covered by countably many Lipschitz hypersurfaces. In particular, the Hausdorff dimension of $\Sigma_{F}$ is at most $N-1$, a result that was originally conjectured by Erdös in [4] (see also [8]).

We now recall a known relation between the family of all connected components of the singular set $\Sigma_{F}$ and the family of the connected components of $\overline{c o} F \backslash F$. For this purpose, let us consider the map $\mathcal{M}: \mathcal{C}(\overline{\mathrm{co}} F \backslash F) \rightarrow \mathcal{P}\left(\Sigma_{F}\right)$ defined by

$$
\mathcal{M}(S)=\Sigma_{F} \cap \operatorname{proj}_{\overline{\mathrm{co}} F}^{-1}(S) \quad \forall S \in \mathcal{C}(\overline{\mathrm{co}} F \backslash F) .
$$

The following result was proved by Veselý in [9].

Lemma 2.2. $\mathcal{M}$ is an injective map with range equal to $\mathcal{C}\left(\Sigma_{F}\right)$.

For a given point $x \notin F$, consider a ball $B(x, r) \subseteq \mathbb{R}^{n} \backslash F$. Clearly, the family

$$
\mathcal{B}(x, r):=\left\{B(y, R): B(x, r) \subseteq B(y, R) \subseteq \mathbb{R}^{n} \backslash F\right\}
$$

is non-empty. If $B(\widehat{x}, \widehat{r})$ is an element of $\mathcal{B}(x, r)$ having maximal radius, then we say that $B(\widehat{x}, \widehat{r})$ is a maximal ball containing $B(x, r)$. Obviously, the radius $\widehat{r}=\widehat{r}(x, r)$ of a maximal ball is a well-defined function of $(x, r)$. On the other hand, the center of a maximal ball need not be unique. Nevertheless, the norm of the center of any maximal ball satisfies the lower bound

$$
\|\widehat{x}\| \geq\left\|\widehat{x}-x_{0}\right\|-\left\|x_{0}\right\| \geq \widehat{r}-\left\|x_{0}\right\|
$$

where $x_{0}$ is a fixed element of $F$.

The next lemma is a usual tool for proving Motzkin's Theorem [6]. Among other things, it ensures the existence of a maximal ball. 
Lemma 2.3. Let $x \in \overline{\mathrm{co}} F \backslash F$, and let $r>0$ be such that $\overline{B(x, r)} \subseteq \mathbb{R}^{n} \backslash F$. Then, the following results hold.

1. There exists a maximal ball containing $B(x, r)$.

2. If $B(\widehat{x}, \widehat{r})$ is a maximal ball containing $B(x, r)$, then $\partial B(\widehat{x}, \widehat{r}) \cap F$ contains at least two different points, and so $\widehat{x} \in \Sigma_{F}$. If, in addition, $x \in \partial(\overline{\operatorname{co}} F) \backslash F$, then

$$
\lim _{r \downarrow 0}\|\widehat{x}\|=\infty .
$$

Proof. We imitate the proof of Motzkin's Theorem given in [5]. First, let us prove that

$$
K:=\left\{(y, R) \in \mathbb{R}^{n} \times\right] 0, \infty[: B(y, R) \in \mathcal{B}(x, r)\}
$$

is compact. For this purpose, we observe that, for any $(y, R) \in \mathbb{R}^{n} \times \mathbb{R}$,

$$
(y, R) \in K \quad \Longleftrightarrow \quad\|y-x\| \leq R-r \quad \text { and } \quad d_{F}(y) \geq R .
$$

In order to show that $K$ is bounded, we note that there exist a point $z \in(\operatorname{co} F \backslash F) \cap$ $B(x, r)$ and a positive number $\varepsilon$ such that $B(z, \varepsilon) \subseteq B(x, r)$. Also, for a suitable choice of points $z_{1}, \ldots, z_{n+1} \in F$, we have that $z \in \operatorname{co}\left\{z_{1}, \ldots, z_{n+1}\right\}$. Set

$$
A=\max \left\{\left\|z-z_{i}\right\|: 1 \leq i \leq n+1\right\} .
$$

Let $(y, R) \in K$. Then

$$
\|y-z\| \leq R-\varepsilon
$$

and

$$
(y-z) \cdot\left(z_{i}-z\right) \geq 0
$$

for some $i \in\{1, \ldots, n+1\}$. Indeed, $(2.5)$ follows observing that $0=(y-z) \cdot(z-z)$ is a convex combination of $\left\{(y-z) \cdot\left(z-z_{i}\right): 1 \leq i \leq n+1\right\}$.

Now, by (2.4) and (2.5),

$$
(R-\varepsilon)^{2} \geq\|z-y\|^{2} \geq\left\|y-z_{i}\right\|^{2}-\left\|z_{i}-z\right\|^{2} \geq R^{2}-A^{2} .
$$

So, $R \leq \frac{\varepsilon^{2}+A^{2}}{2 \varepsilon}$. Hence, (2.3) implies that $K$ is bounded and closed, thus compact. Therefore, a maximal ball does exist in $\mathcal{B}(x, r)$.

For the proof of the fact that $\partial B(\widehat{x}, \widehat{r}) \cap F$ contains at least two different points the reader is referred to [5, p. 62].

Finally, let $x \in \partial(\overline{\operatorname{co}} F) \backslash F$ and fix $r_{0}>0$ so that $\overline{B\left(x, r_{0}\right)} \subseteq \mathbb{R}^{n} \backslash F$. Let $\theta$ be any unit vector in the normal cone to $\overline{\operatorname{co}} F$ at $x$, that is

$$
\|\theta\|=1 \quad \text { and } \quad(y-x) \cdot \theta \leq 0 \quad \forall y \in \overline{\mathrm{co}} F .
$$

We will show that

$$
0<r<r_{0} \Longrightarrow \widehat{r} \geq \frac{r^{2}+r_{0}^{2}}{2 r} .
$$

This, in view of (2.1), will imply the conclusion (2.2). To prove (2.7), let us define $B_{R}:=B(x+(R-r) \theta, R)$ for any $R>r$, and observe that $B(x, r) \subseteq B_{R}$. Then, it suffices to check that

$$
r<R \leq \frac{r^{2}+r_{0}^{2}}{2 r} \Longrightarrow B_{R} \subseteq \mathbb{R}^{n} \backslash F .
$$


Aiming at (2.8), let $z \in B_{R} \cap \overline{\mathrm{co}} F$. Then, $\|z-x-(R-r) \theta\|^{2}<R^{2}$. So, owing to (2.6),

$$
\|z-x\|^{2}<2 r R-r^{2} \leq r_{0}^{2}
$$

provided that $R$ is chosen as in (2.8). Thus, $z \in B\left(x, r_{0}\right) \subseteq \mathbb{R}^{n} \backslash F$ and the proof is complete.

Remark 2.4. We note that a simple consequence of (2.2) above is that $\Sigma_{F}$ is unbounded whenever $\partial(\overline{\mathrm{co}} F) \not \subset F$. This result was originally proved by Motzkin in [7] for $F$ compact.

\section{Connected Components of $\Sigma_{F}$}

The main result of this section is the following characterization of the bounded connected components of $\Sigma_{F}$ in terms of the one-to-one correspondence $\mathcal{M}$ defined in section 2 .

Theorem 3.1. Let $S \in \mathcal{C}(\overline{\mathrm{co}} F \backslash F)$. Then $\mathcal{M}(S)$ is bounded if and only if $S$ is bounded and $S \in \mathcal{C}\left(\mathbb{R}^{n} \backslash F\right)$.

For the proof of Theorem 3.1 we need two simple lemmas.

Lemma 3.2. Any two points $y \in S$ and $x \in \mathcal{M}(S)$ belong to the same connected component of $\mathbb{R}^{n} \backslash F$.

Proof. First, by the very definition of $\mathcal{M}$, we have that $z:=\operatorname{proj}_{\overline{c o}} F(x) \in S$. Moreover, $z \notin F$ as $x$ is singular for $d_{F}$. Thus, $[x, z]$ is contained in $\mathbb{R}^{n} \backslash F$ and so $x$ and $z$ lie in the same connected component of $\mathbb{R}^{n} \backslash F$. Since $y, z \in S$ and $S$ is a connected component of $\overline{\mathrm{co}} F \backslash F$, the conclusion follows.

We recall that the notion of maximal ball was introduced just before the statement of Lemma 2.3.

Lemma 3.3. Let $y \in S$ and suppose $\overline{B(y, r)} \subseteq \mathbb{R}^{n} \backslash F$. If $B(\widehat{y}, \widehat{r})$ is a maximal ball containing $B(y, r)$, then $\widehat{y} \in \mathcal{M}(S)$.

Proof. We have to show that $z:=\operatorname{proj}_{\overline{c o} F}(\widehat{y})$ lies in $S$. For this purpose let us note that $z \in B(\widehat{y}, \widehat{r})$, as any closed maximal ball touches $F$ at two points at least. Hence,

$$
y, z \in B(\widehat{y}, \widehat{r}) \cap \overline{\operatorname{co}} F .
$$

Since $B(\widehat{y}, \widehat{r}) \cap \overline{\mathrm{co}} F$ is a convex set contained in $\overline{\mathrm{co}} F \backslash F$, we conclude that $z \in S$ as required.

The following is a refinement of the result described in Remark 2.4.

Corollary 3.4. If $\partial(\overline{\mathrm{co}} F) \nsubseteq F$, then at least one connected component of $\Sigma_{F}$ is unbounded.

Proof. Take $y \in \partial(\overline{\mathrm{co}} F) \backslash F$ and let $S$ be the connected component of $\overline{\mathrm{co}} F \backslash F$ containing $y$. By $(2.2), \mathcal{M}(S)$ is unbounded.

We are now ready to prove the announced characterization. 
Proof of Theorem 3.1. The proof of the "if" part is easy. Indeed, let $S$ be a bounded connected component of $\mathbb{R}^{n} \backslash F$. Then, by Lemma 3.2, $\mathcal{M}(S) \subseteq S$.

The "only if" part will be proved by contradiction. So, suppose $S$ is not a bounded component of $\mathbb{R}^{n} \backslash F$. We will then show that $\mathcal{M}(S)$ is unbounded. Aiming at this, let us distinguish two cases.

Case 1: $S$ is an unbounded component of $\mathbb{R}^{n} \backslash F$. Let $z \in F$ be fixed. For $y \in S$, let $\overline{B(y, r)} \subseteq \mathbb{R}^{n} \backslash F$. If $B(\widehat{y}, \widehat{r})$ is a maximal ball containing $B(y, r)$, then $\widehat{y} \in \mathcal{M}(S)$ by Lemma 3.3. Also, $\|y-\widehat{y}\| \leq\|z-\widehat{y}\|$ as $z \notin B(\widehat{y}, \widehat{r})$. Thus,

$$
\|\widehat{y}\| \geq\|y\|-\|\widehat{y}-y\| \geq\|y\|-\|\widehat{y}-z\| \geq\|y\|-\|\widehat{y}\|-\|z\| .
$$

Therefore, $\|\widehat{y}\| \geq \frac{1}{2}(\|y\|-\|z\|)$. Since $S$ is unbounded, $\|y\|$ can be taken arbitrarily large. So, $\mathcal{M}(S)$ is unbounded.

Case 2: $S$ is not a component of $\mathbb{R}^{n} \backslash F$. Let $\widetilde{S}$ be the component of $\mathbb{R}^{n} \backslash F$ containing $S$ and fix $x_{0} \in S$. Clearly, $\widetilde{S} \nsubseteq \overline{\mathrm{co}} F$ and so there exists a point $x_{1} \in$ $\widetilde{S} \backslash \overline{\operatorname{co}} F$. Let $\gamma:[0,1] \rightarrow \widetilde{S}$ be a continuous arc such that $\gamma(0)=x_{0}, \gamma(1)=x_{1}$ and define

$$
\bar{t}=\inf \{t \in[0,1]: \gamma(t) \notin \overline{\mathrm{co}} F\} .
$$

Then, $\gamma(\bar{t}) \in \overline{\mathrm{co}} F$ as $\overline{\mathrm{co}} F$ is closed. Furthermore, $\gamma(\bar{t}) \in \partial(\overline{\mathrm{co}} F)$ as a sequence $t_{j} \downarrow \bar{t}$ such that $\gamma\left(t_{j}\right) \notin \overline{\mathrm{co}} F$ does exist. On the other hand, $\gamma(t) \in \overline{\mathrm{co}} F$ for every $t \in[0, \bar{t}]$. Therefore, $\gamma(\bar{t})$ and $x_{0}$ belong to the same component of $\overline{\operatorname{co}} F \backslash F$. In conclusion, $\gamma(\bar{t}) \in S \in \mathcal{C}(\overline{\mathrm{co}} F \backslash F)$ and the proof can be easily completed applying Lemma 3.3 and (2.2).

Remark 3.5. We note that the above proof (Case 2) also shows the following result: if $S \in \mathcal{C}(\overline{\mathrm{co}} F \backslash F)$ is not a connected component of $\mathbb{R}^{n} \backslash F$, then $S \cap \partial(\overline{\mathrm{co}} F)$ is nonempty.

Corollary 3.6. Given $G \in \mathcal{C}\left(\mathbb{R}^{n} \backslash F\right)$, let

$$
\mathcal{R}(G)=\{\mathcal{M}(S): S \in \mathcal{C}(\overline{\mathrm{co}} F \backslash F), S \subseteq G\} .
$$

If $G$ is bounded, then $\mathcal{R}(G)$ has precisely one element, which is bounded. If, on the contrary, $G$ is unbounded, then all elements of $\mathcal{R}(G)$ are unbounded.

Proof. If $G$ is bounded, we have that $G \subseteq \overline{\mathrm{co}} F$ for, by the Hahn-Banach Theorem, any $x \notin \overline{\mathrm{co}} F$ lies in an unbounded component of $\mathbb{R}^{n} \backslash F$. So, $G$ is also a connected component of $\overline{\mathrm{co}} F \backslash F$. If, on the contrary, $G$ is unbounded, then no set $S$ in the definition of $\mathcal{R}(G)$ can be a bounded component of $\mathbb{R}^{n} \backslash F$. The conclusion follows directly from Theorem 3.1.

In particular, we obtain the following.

Corollary 3.7. All connected components of $\Sigma_{F}$ are unbounded if and only if all connected components of $\mathbb{R}^{n} \backslash F$ are unbounded.

We note that, in the above corollary, the singular set $\Sigma_{F}$ may well be empty.

\section{Asymptotic Behaviour of $\Sigma_{F}$}

In this section we are interested in the behaviour at infinity of the unbounded components of the singular set of the distance. Hereafter, we shall assume that $n \geq 2$, the case $n=1$ being trivial. 
Theorem 4.1. Let $F$ be compact and let $C$ be an unbounded connected component of $\Sigma_{F}$. Then there exists a half line $\left\{x_{0}+t v: t \geq 0\right\}$, where $x_{0}, v \in \mathbb{R}^{n}$ and $\|v\|=1$, such that $d_{C}\left(x_{0}+t v\right) \rightarrow 0$ as $t \rightarrow+\infty$.

The proof of the above theorem relies on the following simple property of compact sets.

Lemma 4.2. Let $K \subseteq \mathbb{R}^{n}$ be a compact set satisfying

$$
x_{n} \leq 0 \quad \forall x \in K
$$

and

$$
K_{0}:=\left\{x \in K: x_{n}=0\right\} \neq \emptyset .
$$

Then, for any $R>0$,

$$
\sup _{\|x\| \leq R, x_{n}=0} \sup _{y \in \operatorname{sroj}_{K}\left(x+t e_{n}\right)} d_{K_{0}}(y) \rightarrow 0 \quad \text { as } \quad t \rightarrow+\infty .
$$

Proof. The conclusion will follow if we show that for any $\varepsilon>0$ there exists $\alpha_{\varepsilon}>0$ such that

$$
y \in K, d_{K_{0}}(y) \geq \varepsilon \quad \Rightarrow \quad y_{n} \leq-\alpha_{\varepsilon}
$$

and that, for any $R>0$,

$$
\sup _{\|x\| \leq R, x_{n}=0} \sup _{y \in \operatorname{proj}_{K}\left(x+t e_{n}\right)}\left|y_{n}\right| \rightarrow 0
$$

as $t \rightarrow+\infty$. Also, it suffices prove (4.2) since (4.1) is an immediate consequence of the compactness of $K$.

Let $q \in K_{0}$ be fixed. Then, for any $R>0$, any $x \in \mathbb{R}^{n}$ satisfying $\|x\| \leq R, x_{n}=0$, and any $y \in \operatorname{proj}_{K}\left(x+t e_{n}\right)$ we have that

$$
\left|t-y_{n}\right| \leq\left\|x+t e_{n}-y\right\| \leq\left\|x+t e_{n}-q\right\| \leq \sqrt{\|x-q\|^{2}+t^{2}} \leq \sqrt{(R+\|q\|)^{2}+t^{2}} .
$$

Therefore,

$$
t-\sqrt{(R+\|q\|)^{2}+t^{2}} \leq y_{n} \leq 0
$$

and (4.2) follows as $t \rightarrow+\infty$.

We are now ready to prove the existence of an asymptote.

Proof of Theorem 4.1. Let $S \in \mathcal{C}(\overline{\mathrm{co}} F \backslash F)$ be such that $C=\mathcal{M}(S)$. Since $C$ is unbounded, Theorem 3.1 implies that either $S$ is unbounded or $S \notin \mathcal{C}\left(\mathbb{R}^{n} \backslash F\right)$. On the other hand, $\overline{\mathrm{co}} F \backslash F$ is bounded as $F$ is compact, and this shows that $S$ cannot be a component of $\mathbb{R}^{n} \backslash F$.

Now, in view of Remark 3.5 there exists a point $\bar{x} \in S$ such that $\bar{x} \in \partial(\overline{\operatorname{co}} F) \backslash F$. Let $\Pi$ be a support hyperplane to $\overline{c o} F$ through $\bar{x}$. Possibly changing coordinates, we can and do assume that

$$
\bar{x} \in \Pi=\left\{x \in \mathbb{R}^{n}: x_{n}=0\right\}
$$

and

$$
\overline{\mathrm{co}} F \subseteq\left\{x \in \mathbb{R}^{n}: x_{n} \leq 0\right\}
$$

Let us set

$$
A:=F \cap \Pi \quad \text { and } \quad B:=(\overline{\mathrm{co}} F) \cap \Pi \text {. }
$$


Notice that $B$ is the closed convex hull of $A$. Indeed, the inclusion $\overline{\operatorname{co}} A \subseteq B$ is trivial. Conversely, let $x \in B$. Then, $x_{n}=0$ and there exist $x^{(1)}, \ldots, x^{(k)} \in F$ and $\lambda_{1}, \ldots, \lambda_{k}>0$ such that

$$
x=\sum_{i=1}^{k} \lambda_{i} x^{(i)}, \quad \sum_{i=1}^{k} \lambda_{i}=1 .
$$

Hence, $x_{n}^{(i)}=0$ for any $1 \leq i \leq k$, and so $x^{(1)}, \ldots, x^{(k)} \in A$.

Next, we observe that $\bar{x} \in B \backslash A$ by construction. Applying Lemma 2.2 to the set $A \subseteq \Pi \simeq \mathbb{R}^{n-1}$, we find a point $\bar{z} \in \Pi \cap \Sigma_{A}$ whose projection onto $B$ lies in the same connected component of $B \backslash A$ as $\bar{x}$. Without loss of generality we will assume that $\bar{z}=0$.

Let $R>0$ be such that $B(R) \subseteq \mathbb{R}^{n} \backslash F$. Then, $d_{A}(0) \geq R$. We want to show that for any $0<r<R$ there exists $T(r)>0$ such that, for any $t \geq T(r)$, the translated disc $(B(r) \cap \Pi)+t e_{n}$ contains a singular point $x(t) \in \Sigma_{F}$. We will split our reasoning into four steps.

Step 1: we claim that, for any $0<r<R$, there exists a number $\sigma_{r}>0$ such that

$$
d_{A}(x)<r-\sigma_{r}+d_{A}(0) \quad \forall x \in \Pi \cap \partial B(r) .
$$

Indeed, let $p, p^{\prime} \in A\left(p \neq p^{\prime}\right)$ be such that

$$
\|p\|=d_{A}(0)=\left\|p^{\prime}\right\| \text {. }
$$

For any $x \in \Pi \cap \partial B(r)$ we have that either $0 \notin[x, p]$ or $0 \notin\left[x, p^{\prime}\right]$. Assume that $0 \notin[x, p]$. Then,

$$
d_{A}(x) \leq\|p-x\|<\|p\|+\|x\|=d_{A}(0)+r .
$$

A compactness argument yields (4.3).

Step 2: for $0<r<R$ and $t \geq 0$ let us define

$$
\alpha_{r}(x)=\operatorname{proj}_{\Pi \cap \overline{B(r)}}(x), \quad x \in \mathbb{R}^{n},
$$

and

$$
\phi_{t}(x)=\operatorname{proj}_{F}\left(x+t e_{n}\right), \quad x \in \Pi \cap \overline{B(r)} .
$$

Notice that $\alpha_{r}$ is, in fact, single-valued and continuous. Moreover,

$$
\alpha_{r}=\alpha_{r} \circ \operatorname{proj}_{\Pi}
$$

Map $\phi_{t}$, on the contrary, is set-valued in general. Let us denote by $\Phi$ the set of all $t \geq 0$ such that $\phi_{t}$ is single-valued. Then, the product map

$$
\beta_{r, t}:=\alpha_{r} \circ \phi_{t}: \Pi \cap \overline{B(r)} \rightarrow \Pi \cap \overline{B(r)}
$$

is well-defined and continuous for any $0<r<R$ and $t \in \Phi$.

Step 3: let $r \in] 0, R\left[\right.$ be fixed and set $\rho:=\min \left\{R-r, \sigma_{r}\right\}$, with $\sigma_{r}$ given by Step 1. Then, by Lemma 4.2, there exists $T(r)>0$ such that

$$
d_{A}\left(\phi_{t}(x)\right)<\rho \quad \forall x \in \Pi \cap \overline{B(r)}
$$

for every $t \in \Phi$ satisfying $t \geq T(r)$. We claim that, for such values of $t$,

$$
\beta_{r, t}(x) \in \partial B(r) \cap \Pi \quad \forall x \in \Pi \cap \overline{B(r)}
$$

and

$$
\beta_{r, t}(x) \neq-x \quad \forall x \in \Pi \cap \partial B(r) .
$$


To prove our first claim, let $x \in \Pi \cap \overline{B(r)}$ be fixed. In view of (4.4) we have that, for some $y \in A,\left\|y-\phi_{t}(x)\right\|<\rho$. Set

$$
x(t):=\operatorname{proj}_{\Pi}\left(\phi_{t}(x)\right) .
$$

Then, $\|y-x(t)\| \leq\left\|y-\phi_{t}(x)\right\|<\rho$, and so

$$
\|x(t)\|>\|y\|-\rho \geq R-\rho \geq r .
$$

Inclusion (4.5) easily follows.

We now turn to the proof of (4.6). Suppose that a point $x$ exists on the sphere $\Pi \cap \partial B(r)$ such that $\beta_{r, t}(x)=-x$. We will show that this gives a contradiction. In fact, since $x(t)$ is a negative multiple of $x$, using (4.8) we have that

$$
\|x-x(t)\|=r+\|x(t)\| \geq r+\|y\|-\rho \geq r+d_{A}(0)-\sigma_{r}>0 .
$$

Therefore, recalling (4.7),

$$
\left\|x+t e_{n}-\phi_{t}(x)\right\| \geq \sqrt{\|x-x(t)\|^{2}+t^{2}} \geq \sqrt{\left(r+d_{A}(0)-\sigma_{r}\right)^{2}+t^{2}} .
$$

On the other hand, owing to (4.3) we can find a point $y^{\prime} \in A$ such that

$$
\left\|x+t e_{n}-y^{\prime}\right\|<\sqrt{\left(r+d_{A}(0)-\sigma_{r}\right)^{2}+t^{2}} .
$$

Since (4.9) and (4.10) contradict the definition of $\phi_{t}$, the proof of (4.6) is complete.

Step 4: we claim that, if $t \geq T(r)$, then $t \notin \Phi$. In other words, we will show that the projection map $\phi_{t}$ must be multi-valued at some point for sufficiently large $t$. For suppose not. Then, on account of (4.5) and (4.6), the continuous map $-\beta_{r, t}$ would have no fixed points in $\Pi \cap \overline{B(r)}$, contrary to Brouwer's Theorem. Hence, our claim follows.

From Steps 1 to 4 above we conclude that, for any $r>0$, there exists $T(r)>0$ such that, for any $t \geq T(r)$, a singular point $\xi(t) \in \Sigma_{F}$ exists so that $\left\|\xi(t)-t e_{n}\right\| \leq r$. In order to complete the proof, it remains to show that $\xi(t)$ belongs to the connected component $C$ of $\Sigma_{F}$, that we fixed at the beginning. This follows from our next lemma.

Lemma 4.3. Let $S, \bar{x}, \Pi, e_{n}$ be defined as in the proof of Theorem 4.1. Then there exist $r_{0}, T_{0}>0$ such that, for any $t \geq T_{0}$,

$$
\operatorname{proj}_{\overline{\mathrm{co}} F}\left(x+t e_{n}\right) \in S \quad \forall x \in \Pi \cap \overline{B\left(r_{0}\right)} .
$$

Proof. First, without loss of generality, we will assume that

$$
\bar{x}=\operatorname{proj}_{B}(0) .
$$

In fact, since $\bar{x}$ and $\operatorname{proj}_{B}(0)$ belong to the same connected component of $B \backslash A$, they belong to the same component of $\overline{\mathrm{co}} F \backslash F$ as well.

Now, let $B(\bar{x}, \rho) \subseteq \mathbb{R}^{n} \backslash F$. Then,

$$
B(\bar{x}, \rho) \cap \overline{c o} F \subseteq S .
$$

Therefore, in view of (4.11), a compactness argument shows that

$$
\|y\| \geq\|\bar{x}\|+\sigma \quad \forall y \in B \backslash B(\bar{x}, \rho)
$$

for some $\sigma>0$. Furthermore, by a continuity argument we conclude that, for some $\eta>0$, all points $y \in \overline{\mathrm{co}} F \backslash B(\bar{x}, \rho)$, with $y_{n} \geq-\eta$, satisfy the estimate below

$$
\left\|\operatorname{proj}_{\Pi}(y)\right\| \geq\|\bar{x}\|+\frac{\sigma}{2} \text {. }
$$


By another continuity argument, the last inequality can be easily refined as follows: there exists $r_{0}>0$ such that

$$
\left\|\operatorname{proj}_{\Pi}(y)-x\right\|>\|\bar{x}-x\| \quad \forall x \in \Pi \cap \overline{B\left(r_{0}\right)}
$$

for any $y \in \overline{\operatorname{co}} F \backslash B(\bar{x}, \rho)$ satisfying $y_{n} \geq-\eta$.

Next, by Lemma 4.2 applied to $K=\overline{\mathrm{co}} F$ we deduce that, if $t$ is sufficiently large, say $t \geq T_{0}$, then

$$
e_{n} \cdot \operatorname{proj}_{\overline{\mathrm{co}} F}\left(x+t e_{n}\right) \geq-\eta \quad \forall x \in \Pi \cap \overline{B\left(r_{0}\right)}
$$

where $\eta>0$ is fixed as in (4.13).

To complete the proof of the lemma, in view of (4.12), it suffices to show that, for $t \geq T_{0}$,

$$
\operatorname{proj}_{\overline{\mathrm{co}} F}\left(x+t e_{n}\right) \in B(\bar{x}, \rho) \quad \forall x \in \Pi \cap \overline{B\left(r_{0}\right)} .
$$

We now proceed to check (4.15). Let $t \geq T_{0}$ and $x \in \Pi \cap \overline{B\left(r_{0}\right)}$ be fixed, and set $z:=\operatorname{proj}_{\overline{c o} F}\left(x+t e_{n}\right)$. Suppose $z \notin B(\bar{x}, \rho)$. Then, owing to (4.13) and (4.14), we have

$$
\left\|\operatorname{proj}_{\Pi}\left(x+t e_{n}-z\right)\right\|=\left\|x-\operatorname{proj}_{\Pi}(z)\right\|>\|\bar{x}-x\|=\left\|\operatorname{proj}_{\Pi}\left(x+t e_{n}-\bar{x}\right)\right\| .
$$

Moreover, $\left|\left(x+t e_{n}-z\right)_{n}\right| \geq t=\left|\left(x+t e_{n}-\bar{x}\right)_{n}\right|$. So,

$$
\left\|x+t e_{n}-z\right\|>\left\|x+t e_{n}-\bar{x}\right\|
$$

in contrast with the definition of $z$.

\section{Asymptotic Behaviour in Dimension Two}

In this last section we shall restrict our attention to the two-dimensional case for which stronger results can be obtained. Let us recall that Theorem 4.1 ensures the existence of a half line in $\mathbb{R}^{n}$, asymptotic to any unbounded connected component, $C$, of $\Sigma_{F}$. However, from the proof of this theorem it actually follows that there exists $\phi:\left[0,+\infty\left[\rightarrow v^{\perp}\right.\right.$, with $\phi(t) \rightarrow 0$ as $t \rightarrow+\infty$, such that $x_{0}+t v+\phi(t) \in C$. As we will see next, when $n=2$ one can show that $\phi$ is continuous.

Theorem 5.1. Let $F \subseteq \mathbb{R}^{2}$ be a compact set, and $C$ be an unbounded connected component of $\Sigma_{F}$. Then there exists a half line $\left\{x_{0}+t v: t \geq 0\right\}$, where $x_{0}, v \in \mathbb{R}^{2}$ and $\|v\|=1$, and a continuous map $\phi:\left[0,+\infty\left[\rightarrow v^{\perp}\right.\right.$ such that

$$
x_{0}+t v+\phi(t) \in C \quad \text { and } \quad \phi(t) \rightarrow 0 \quad \text { as } t \rightarrow+\infty .
$$

Proof. We shall use the same notation as in the proof of Theorem 4.1. Notice that, in this special case, $\Pi$ is a straight line, $0 \in \Pi \cap \Sigma_{A}$, and $\operatorname{proj}_{A}(0)=\{(a, 0),(-a, 0)\}$ for some $a>0$.

Let $0<r<a$. By Lemma 4.2 we deduce that there exists $\tau(r)>0$ such that, for any $t \geq \tau(r)$ and any $s \in[-r, r]$,

$$
d_{A}(y)<a-r \quad \forall y \in \operatorname{proj}_{F}\left(s e_{1}+t e_{2}\right) .
$$

Hence, for all such values of $t$ and $s$,

$$
\operatorname{proj}_{F}\left(s e_{1}+t e_{2}\right) \subseteq F_{1} \cup F_{2}
$$

where

$$
F_{1}:=\left\{x \in F: x_{1} \leq-r\right\}, \quad F_{2}:=\left\{x \in F: x_{1} \geq r\right\} .
$$


Therefore,

$$
d_{F_{1}}\left(s e_{1}+t e_{2}\right)=d_{F_{2}}\left(s e_{1}+t e_{2}\right) \quad \Longrightarrow \quad s e_{1}+t e_{2} \in \Sigma_{F}
$$

for any $t \geq \tau(r)$ and any $s \in[-r, r]$.

Now, let us define

$$
f_{t}(s)=d_{F_{1}}\left(s e_{1}+t e_{2}\right)-d_{F_{2}}\left(s e_{1}+t e_{2}\right) \quad(t \geq \tau(r),-r \leq s \leq r) .
$$

We claim that, if $t$ is sufficiently large, say $t \geq T(r) \geq \tau(r)$,

(i) $f_{t}$ is strictly increasing;

(ii) $f_{t}(-r)<0, f_{t}(r)>0$.

Indeed, let $-r \leq s_{1}<s_{2} \leq r$ and let $y \in \operatorname{proj}_{F_{1}}\left(s_{2} e_{1}+t e_{2}\right)$. Then

$$
d_{F_{1}}\left(s_{1} e_{1}+t e_{2}\right) \leq\left\|s_{1} e_{1}+t e_{2}-y\right\|<\left\|s_{2} e_{1}+t e_{2}-y\right\|=d_{F_{1}}\left(s_{2} e_{1}+t e_{2}\right) .
$$

Similarly, $d_{F_{2}}\left(s_{1} e_{1}+t e_{2}\right)>d_{F_{2}}\left(s_{2} e_{1}+t e_{2}\right)$. So, our claim (i) follows.

Next, to check that $f_{t}(r)>0$, we can apply Lemma 4.2 to prove the existence of a number $T(r) \geq \tau(r)$ such that

$$
d_{F_{1} \cap \Pi}(y)<r \quad \forall y \in \operatorname{proj}_{F_{1}}\left(s e_{1}+t e_{2}\right) \quad \forall s \in[-r, r]
$$

for every $t \geq T(r)$. Also, observe that $x_{1} \leq-a$ for any $x \in F_{1} \cap \Pi$. Thus,

$$
y_{1} \leq r-a \quad \forall y \in \operatorname{proj}_{F_{1}}\left(s e_{1}+t e_{2}\right) \quad \forall s \in[-r, r] .
$$

Consequently,

$$
\left\|r e_{1}+t e_{2}-y\right\|=\sqrt{\left(r-y_{1}\right)^{2}+\left(t-y_{2}\right)^{2}} \geq \sqrt{a^{2}+t^{2}}
$$

for any $y \in \operatorname{proj}_{F_{1}}\left(r e_{1}+t e_{2}\right)$ and any $t \geq T(r)$. Therefore,

$$
\begin{aligned}
d_{F_{1}}\left(r e_{1}+t e_{2}\right) & \geq \sqrt{a^{2}+t^{2}}>\sqrt{(a-r)^{2}+t^{2}} \\
& =\left\|r e_{1}+t e_{2}-(a, 0)\right\| \geq d_{F_{2}}\left(r e_{1}+t e_{2}\right)
\end{aligned}
$$

and so $f_{t}(r)>0$, for any $t \geq T(r)$. Since the inequality $f_{t}(-r)<0$ can be obtained similarly, the proof of (ii) is complete.

In conclusion, from properties (i) and (ii) above we deduce that, for any $t \geq T(r)$, the function $f_{t}$ has a unique zero in $[-r, r]$, say $s(t)$. Moreover, as one can easily check, $s(t)$ depends continuously on $t$. In view of (5.2), we have thus shown that for any $r \in] 0, a[$ there exists $T(r)>0$ such that

$$
s(t) e_{1}+t e_{2} \in \Sigma_{F} \quad \forall t \geq T(r) .
$$

To construct the desired map, it suffices to fix $\left.r=r_{0} \in\right] 0, a[$ and take

$$
x_{0}=T\left(r_{0}\right) e_{2}, \quad v=e_{2}, \quad \phi(t)=s\left(t+T\left(r_{0}\right)\right) e_{1} .
$$

The fact that $x_{0}+t v+\phi(t) \in C$ follows from Lemma 4.3.

Remark 5.2. In the sequel, we will need a property that we have derived, incidentally, in the proof of Theorem 5.1, namely that $\operatorname{proj}_{F}\left(x_{0}+t v+\phi(t)\right)$ tends to the set $\{(a, 0),(-a, 0)\}$ as $t \rightarrow+\infty$. More precisely, we claim that the distance of $(a, 0)$ from $\operatorname{proj}_{F_{2}}\left(x_{0}+t v+\phi(t)\right)$ tends to 0 as $t \rightarrow+\infty$, and so does the distance of $(-a, 0)$ from $\operatorname{proj}_{F_{1}}\left(x_{0}+t v+\phi(t)\right)$. In fact, by (5.1), for any $t \geq \tau(r), s \in[-r, r]$, and any $x \in \operatorname{proj}_{F}\left(s e_{1}+t e_{2}\right)$, we have that $\left|x_{1}\right| \geq r$. Also, $\left|x_{1}\right| \leq a$, for otherwise either $(a, 0)$ or $(-a, 0)$ would be closer than $x$ to $s e_{1}+t e_{2}$. Since, in view of Lemma 4.2 , the $e_{2}-$ component of any $x \in \operatorname{proj}_{F}\left(s e_{1}+t e_{2}\right)$ tends to 0 as $t \rightarrow+\infty$, the claimed property follows. 
Theorem 5.1 ensures that, if $n=2$, then any unbounded connected component of $\Sigma_{F}$ contains at least a continuous arc that propagates up to $\infty$. On the other hand, it is known that any point $\bar{x} \in \Sigma_{F}$ satisfying $\bar{x} \notin \overline{\mathrm{co}} F$ is the initial point of a Lipschitz singular $\operatorname{arc} \xi(\cdot)$ for $d_{F}$, see [3] for the case of $n=2$ and [1] for general $n$. Moreover, $\xi(\cdot)$ is the unique absolutely continuous solution of the problem

$$
\left\{\begin{array}{l}
\xi^{\prime}(s) \in D^{+} d_{F}(\xi(s)), \quad s \in[0, \sigma] \quad \text { a.e. }, \\
\xi(0)=\bar{x}
\end{array}\right.
$$

for some $\sigma>0$. Here, $D^{+} d_{F}(x)$ denotes the superdifferential of $d_{F}$ at $x$, which in this case can also be represented as

$$
D^{+} d_{F}(x)=\frac{x-\operatorname{co}\left(\operatorname{proj}_{F}(x)\right)}{d_{F}(x)} \quad(x \notin F) .
$$

Notice that, in particular, $D^{+} d_{F}(x) \subseteq \bar{B}_{1}$, so that the solution $\xi$ of (5.3) is Lipschitz continuous with constant equal to 1 .

It is then natural to ask whether the two singular arcs above-i.e. the one given by Theorem 5.1 and the solution of problem (5.3) - coincide up to reparametrization. The following result is intended to answer such a question.

Theorem 5.3. Let $F \subseteq \mathbb{R}^{2}$ be a compact set, let $C$ be an unbounded connected component of $\Sigma_{F}$, and let

$$
\gamma(t):=x_{0}+t v+\phi(t) \quad(t \geq 0)
$$

be the continuous singular arc given by Theorem 5.1. Then there exists $\bar{t} \geq 0$ such that the solution $\xi$ of problem (5.3) with $\bar{x}=\gamma(\bar{t})$ is defined for any $s \geq 0$ and coincides with $\gamma(t), t \geq \bar{t}$, up to reparametrization.

Proof. We shall use the same notation as in the proof of Theorem 5.1. In particular, we assume, without loss of generality, that $x \cdot e_{2} \leq 0$ for all $x \in F$. Moreover, having set $A=\left\{x \in F: x \cdot e_{2}=0\right\}$, we suppose that $\operatorname{proj}_{A}(0)=\{(a, 0),(-a, 0)\}$ for some $a>0$. Notice that $\gamma(t)=s(t+T(r)) e_{1}+(t+T(r)) e_{2}$, where $\left.r \in\right] 0, a[$ is fixed and $|s(t)|<a$. We also define sets $F_{1}, F_{2}$ as

$$
F_{1}:=\left\{x \in F: x_{1} \leq-r\right\}, \quad F_{2}:=\left\{x \in F: x_{1} \geq r\right\} .
$$

To begin the proof we note that, in view of Remark 5.2, for sufficiently large $t$, say $t \geq \bar{t}$, we have that

$$
\operatorname{proj}_{F_{1}}(\gamma(t)) \subseteq B_{\frac{a}{3}}(-a, 0) \quad \text { and } \quad \operatorname{proj}_{F_{2}}(\gamma(t)) \subseteq B_{\frac{a}{3}}(a, 0) .
$$

Let $\xi$ be the solution of problem (5.3) with $\bar{x}=\gamma(\bar{t})$. For technical reasons, we will assume - without loss of generality - that $\bar{t} \geq a$.

The core of the proof will consist in showing that, for some $\delta \in] 0, \sigma]$,

$$
\begin{gathered}
d_{F_{1}}(\xi(s))=d_{F_{2}}(\xi(s)) \quad \forall s \in[0, \delta], \\
\left|\xi_{1}(s)\right| \leq r \quad \forall s \in[0, \delta]
\end{gathered}
$$

and

$$
\xi_{2}^{\prime}(s) \geq \frac{1}{2}, \quad s \in[0, \delta] \quad \text { a.e. }
$$

To check the above claims, we note first that the proof of Theorem 5.1 actually shows that $\left|\gamma_{1}(\bar{t})\right|=\left|\xi_{1}(0)\right|<r$ provided that $\delta$ above is properly chosen. Thus, estimate (5.6) follows. 
Next, to check (5.7), we observe that, for any $s \in[0, \delta]$ satisfying the inclusion in $(5.3)$, we have that

$$
\xi^{\prime}(s)=\frac{\xi(s)-w(s)}{d_{F}(\xi(s))},
$$

where $w(s) \in \operatorname{co}\left(\operatorname{proj}_{F}(\xi(s))\right)$. Hence,

$$
\xi_{2}^{\prime}(s)=\frac{\xi_{2}(s)-w_{2}(s)}{d_{F}(\xi(s))} \geq \frac{\xi_{2}(s)}{d_{F}(\xi(s))} \quad \text { a.e. in } \quad[0, \delta] .
$$

Recalling that, on account of $(5.6),\left|\xi_{1}(s)\right| \leq a$, we conclude that

$$
d_{F}(\xi(s)) \leq \min \{\|\xi(s)-(a, 0)\|,\|\xi(s)-(-a, 0)\|\} \leq \sqrt{\xi_{2}^{2}(s)+a^{2}} .
$$

Hence,

$$
\xi_{2}^{\prime}(s) \geq \frac{\xi_{2}(s)}{\sqrt{\xi_{2}^{2}(s)+a^{2}}}=: g(s) \quad \text { a.e. in } \quad[0, \delta] .
$$

Now, $\xi_{2}(0) \geq \bar{t} \geq a$ as $\xi(0)=(\bar{t}+T(r)) e_{2}+s(\bar{t}+T(r)) e_{1}$. So, $g(0) \geq 1 / \sqrt{2}$. Since $g$ is continuous, we conclude that (5.7) is satisfied possibly reducing $\delta>0$.

We now turn to the proof of (5.5). Let us set $\alpha_{i}(s)=d_{F_{i}}(\xi(s))$, for $i=1,2$. Then, it suffices to show that

$$
\left(\alpha_{1}(s)-\alpha_{2}(s)\right)\left(\alpha_{1}^{\prime}(s)-\alpha_{2}^{\prime}(s)\right) \leq 0
$$

for every $s \in] 0, \delta\left[\right.$ at which $\xi, \alpha_{1}, \alpha_{2}$ are differentiable, and inclusion (5.3) is satisfied. Indeed, from (5.9) we deduce that the function $\left(\alpha_{1}(s)-\alpha_{2}(s)\right)^{2}$ has nonpositive derivative a.e. in $[0, \delta]$. Thus, it is decreasing. Since it takes the value 0 at 0 , it vanishes identically in $[0, \delta]$. We now proceed to prove (5.9). Let $s$ be as above, and suppose $\alpha_{2}(s)<\alpha_{1}(s)$. Then, thanks to (5.6), (5.7) and (5.1),

$$
\operatorname{proj}_{F}(\xi(s))=\operatorname{proj}_{F_{2}}(\xi(s)) .
$$

Now, fix $x_{s, i} \in \operatorname{proj}_{F_{i}}(\xi(s))$. By Lemma 2.1 we have

$$
\alpha_{i}^{\prime}(s)=\frac{\xi^{\prime}(s) \cdot\left(\xi(s)-x_{s, i}\right)}{\alpha_{i}(s)} .
$$

Hence, by (5.8),

$$
\begin{aligned}
\alpha_{i}^{\prime}(s) & =\frac{(\xi(s)-w(s)) \cdot\left(\xi(s)-x_{s, i}\right)}{\alpha_{i}(s) d_{F}(\xi(s))} \\
& =\frac{\|\xi(s)-w(s)\|^{2}+\alpha_{i}(s)^{2}-\left\|w(s)-x_{s, i}\right\|^{2}}{2 \alpha_{i}(s) d_{F}(\xi(s))} .
\end{aligned}
$$

By (5.4) and the upper semicontinuity of the projection on a closed set we have, possibly taking a smaller $\delta>0$,

$$
\operatorname{proj}_{F_{1}}(\xi(s)) \subseteq B_{\frac{a}{3}}(-a, 0) \quad \text { and } \quad \operatorname{proj}_{F_{2}}(\xi(s)) \subseteq B_{\frac{a}{3}}(a, 0) .
$$

Therefore, $\left\|w(s)-x_{s, 2}\right\| \leq \frac{2}{3} a$, as $x_{s, 2} \in \operatorname{proj}_{F_{2}}(\xi(s))$ and $w(s) \in \operatorname{co}\left(\operatorname{proj}_{F_{2}}(\xi(s))\right)$ in view of (5.10). By similar considerations, $\left\|w(s)-x_{s, 1}\right\| \geq \frac{4}{3} a$. Since $\alpha_{1}(0)=$ $\alpha_{2}(0)$, as $\xi(0) \in \operatorname{Im} \gamma$, (5.11) yields $\alpha_{2}^{\prime}(s)>\alpha_{1}^{\prime}(s)$ for $0<s<\delta$, provided $\delta$ is sufficiently small. The proof of (5.9) is complete in the case $\alpha_{2}(s)<\alpha_{1}(s)$. The opposite case $\alpha_{2}(s)<\alpha_{1}(s)$, can be treated similarly. We have thus proved (5.5).

Now, let $\xi:\left[0, \tau\left[\rightarrow \mathbb{R}^{2}\right.\right.$ be the maximal solution of problem $(5.3)$, with $\bar{x}=\gamma(\bar{t})$, satisfying (5.5), (5.6) and (5.7). We claim that $\tau=+\infty$, which yields in turn the 


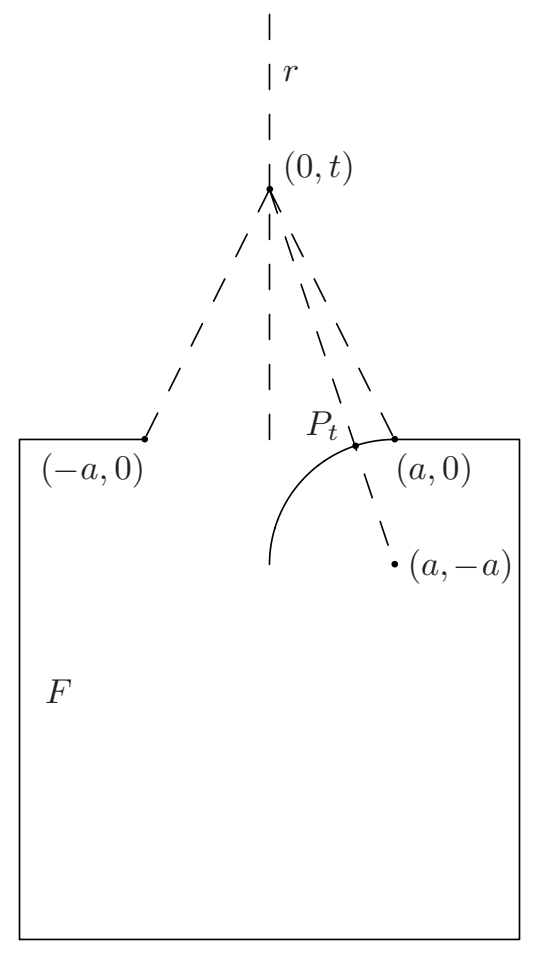

Figure. Here, $P_{t}=\operatorname{proj}_{F}(0, t)$

conclusion of the theorem. For suppose $\tau<+\infty$. Then, owing to (5.5) and (5.6), $\xi\left(\tau^{-}\right):=\lim _{t \uparrow \tau} \xi(t) \in \gamma\left(\left[\bar{t},+\infty[)\right.\right.$. So, we can solve problem (5.3) with $\bar{x}=\xi\left(\tau^{-}\right)$, obtaining a contradiction with the maximality of $\xi$.

We conclude the paper with an example showing that an unbounded component of $\Sigma_{F}$ may well possess an asymptote no point of which belongs to $\Sigma_{F}$.

Example 5.4. Let $F$ be the compact subset of $\mathbb{R}^{2}$ pictured in the Figure, the round part of which consists of an arc of circle of radius $a$, centered at $(a,-a)$

From the proof of Theorem 5.1 it follows that the asymptote of the unbounded connected component of $\Sigma_{F}$ (which in fact coincides with $\Sigma_{F}$ itself) is given by the half-line $r=\{(0, t): t \geq 0\}$. On the other hand, a simple geometric argument shows that $\Sigma_{F} \cap r=\emptyset$. Indeed, the projection onto $F$ of any point $(0, t) \in r$ is given by the intersection $P_{t}$ of $F$ with the straight line through $(0, t)$ and $(a,-a)$.

\section{REFERENCES}

[1] P. Albano and P. Cannarsa, Propagation of Singularities for Solutions of Nonlinear First Order PDEs, Submitted.

[2] G. Alberti, L. Ambrosio and P. Cannarsa, On the singularities of convex functions, Manuscripta Math. 76 (1992), 421-435. MR 94c:26017

[3] K. Bartke and H. Berens, Eine Beschreibung der Nichteindeutigkeitsmenge für die beste Approximation in der Euklidischen Ebene, J. Approx. Theory 47 (1986), 54-74. MR 87j:41069

[4] P. Erdös, Some remarks on the measurability of certain sets, Bull. Amer. Math. Soc. 51 (1945), 728-731. MR 7:197f

[5] L. Hörmander, Notions of convexity, Birkhäuser, Boston, MA, 1994. MR 95k:00002 
[6] T. Motzkin, Sur quelques propriétés caractéristiques des ensembles convexes, Atti Accad. Naz. Lincei Rend. Cl. Sci. Fis. Mat. Natur. 21 (1935), 562-567.

[7] T. Motzkin, Sur quelques propriétés caractéristiques des ensembles bornés non convexes, Atti Accad. Naz. Lincei Rend. Cl. Sci. Fis. Mat. Natur. 21 (1935), 773-779.

[8] C. Pauc, Considérations sur la surface répresentative de la distance d'un point à un ensamble ponctuel plan, Rev. Sci. 77 (1939), 493-496. MR 1:109e

[9] L. Veselý, A connectedness property of maximal monotone operators and its application to approximation theory, Proc. Amer. Math. Soc. 115 (1992), 663-667. MR 92i:47064

[10] U. Westphal and J. Frerking, On a property of metric projections onto closed subsets of Hilbert spaces, Proc. Amer. Math. Soc. 105 (1989), 644-651. MR 89j:41051

[11] L. Zajíček, On the differentiation of convex functions in finite and infinite dimensional spaces, Czechoslovak Math. J. 29 (1979), 340-348. MR 80k:46063

Dipartimento di Matematica, Universitì di Roma "Tor Vergata", Via della Ricerca Scientifica, 00133 Roma (Italy)

E-mail address: cannarsa@ mat.uniroma2.it

Dipartimento di Matematica, Università di Roma "Tor Vergata", Via della Ricerca Scientifica, 00133 Roma (Italy)

E-mail address: peirone@ mat.uniroma2.it 